\title{
Outlook for potential third-generation immersion fluids
}

\author{
Juan López-Gejo ${ }^{a^{*}}$, Joy T. Kunjappu ${ }^{\mathrm{a}}$, J. Zhou ${ }^{\mathrm{b}}$, B. W. Smith ${ }^{\mathrm{b}}$, Paul Zimmerman ${ }^{\mathrm{c}}$, Will Conley ${ }^{\mathrm{d}}$, \\ Nicholas J. Turro ${ }^{\mathrm{a}}$ \\ ${ }^{a}$ Department of Chemistry, Columbia University, New York, NY 10027, USA \\ ${ }^{\mathrm{b}}$ Center for Nanolithography Research, Rochester Institute of Technology, 82 Lomb. Memorial \\ Drive, Rochester, NY, USA \\ ${ }^{c}$ SEMATECH Austin, TX 78741, USA \\ ${ }^{\mathrm{d}}$ Freescale Semiconductor, Centre de Recherche 870 rue Jean Monnet, Crolles, France
}

\begin{abstract}
In a search for alkane candidates for $193 \mathrm{~nm}$ immersion fluids, several alkanes and cycloalkanes were synthesized, purified and screened to ascertain their absorption at $193 \mathrm{~nm}$, refractive index and temperature dispersion coefficient in the context of the actual application. In general, cycloalkanes, and more specifically polycycloalkanes, possess a higher refractive index than do linear alkanes. Decalin, cyclodecane, perhydrophenanthreme (PHP), perhydrofluorene (PHF) and perhydropyrene (PHPY) are examined as potential second and third generation immersion fluids. The use of perhydropyrene, which possesses a high refractive index of 1.7014 at $193 \mathrm{~nm}$, may be limited as an immersion fluid because of high absorption at $193 \mathrm{~nm}$. Mixtures of cycloalkanes can lead to a higher enhancement of the refractive index together with a decrease on the viscosity. Exhaustive purification of the fluids is a critical step in determining the real absorption of the different fluids at $193 \mathrm{~nm}$. Two simple purification processes of these cycloalkanes were developed that led to low absorption fluids in the VUV region. The possibility of forming the oxygen complex in aerated fluids was reduced by purging samples with argon or nitrogen. This easy elimination of the oxygen complex shows the weak bonding nature of this complex.
\end{abstract}

Keywords: Alkanes, third generation immersion fluids, Immersion Microlithography, oxygen complex, refractive index, Absorption $193 \mathrm{~nm}$.

\section{INTRODUCTION}

A current holy grail in optical lithography is the development of novel materials and processes so as to push it toward dimensions less than $32 \mathrm{~nm}$. The minimum feature that can eventually be printed with an optical lithography system is determined by the Rayleigh expression, Eq. 1.1

$$
\mathrm{hp}=\frac{k_{1} \lambda}{N A}
$$

where hp is the $1: 1$ half pitch feature size, $\lambda$ is the lithography wavelength $(193 \mathrm{~nm}), \mathrm{k} 1$ is a measure of the lithography process capability and NA is the numerical aperture of the stepper's lens. NA can be defined as given by Eq. 2

$$
\mathrm{NA}=n_{I F} \sin \theta_{\max }
$$

where $\mathrm{nIF}$ is the immersion fluid's index of refraction at the lithographic wavelength $(193 \mathrm{~nm})$ and $\theta$ the aperture angle, which is the angle sustained by the ray of the largest spatial frequency permitted by the optical system. Therefore, half pitch (hp) can be decreased by decreasing $\lambda$ or increasing the nIF. As the challenges of shorter wavelength (157 nm) became increasingly difficult, usage of immersion based $193 \mathrm{~nm}$ lithography systems became more attractive. ${ }^{2}$ Therefore, for current $193 \mathrm{~nm}$ photolithography systems, there is a need to develop new immersion fluids that have a higher refractive index than water $\mathrm{n}=1.44 .^{3}$ In addition, candidates must have an acceptable transparency at $193 \mathrm{~nm}$. Recently, second generation fluids with a refractive index of $\sim 1.6$ at $193 \mathrm{~nm}$ have been prepared, driven by the growing interest in this area. ${ }^{4,5}$ However, the developed fluids so far still present limitations such as high absorbance $(>0.4$

Advances in Resist Materials and Processing Technology XXIV, edited by Qinghuang Lin Proc. of SPIE Vol. 6519, 651921, (2007) · 0277-786X/07/\$18 · doi: 10.1117/12.695536 
$/ \mathrm{cm})$ at the working wavelength, ${ }^{4}$ or high viscosity. ${ }^{5}$ Recently, alkanes, base second generation fluids have been presented by some manufactures. In spite of the fact that the real chemical composition has not been given by the companies, it is assume that the fluids are decalin or decalin derivatives base fluids. In this work, a critical evaluation of alkanes is presented. Synthesis, purification and characterization have been made for several alkanes, and the limitation in terms of refractive index, and absorption has been established.

From a search of the literature, ${ }^{7}$ we have discovered an excellent linear correlation between the index of refraction (at $589 \mathrm{~nm}$ ) of pure liquid alkanes and liquid cycloalkanes and their densities (Figure 1). The first important conclusion from the correlation is that the higher the density of the alkane or cycloalkane, the higher the refractive index at $589 \mathrm{~nm}$. We assume that knowledge of the value of the refractive index at $589 \mathrm{~nm}$ can reliably be extrapolated to the value at 193 $\mathrm{nm}$. We start with the working hypothesis that "electron density" and "free volume" will be useful concepts for a theoretical understanding of the correlation of chemical structure and refractive index. The hypothesis interprets the correlation, shown in Figure 1, to mean that liquids with small free volume, which correspond to high liquid density, will correlate with a high electronic density and high refractive index.

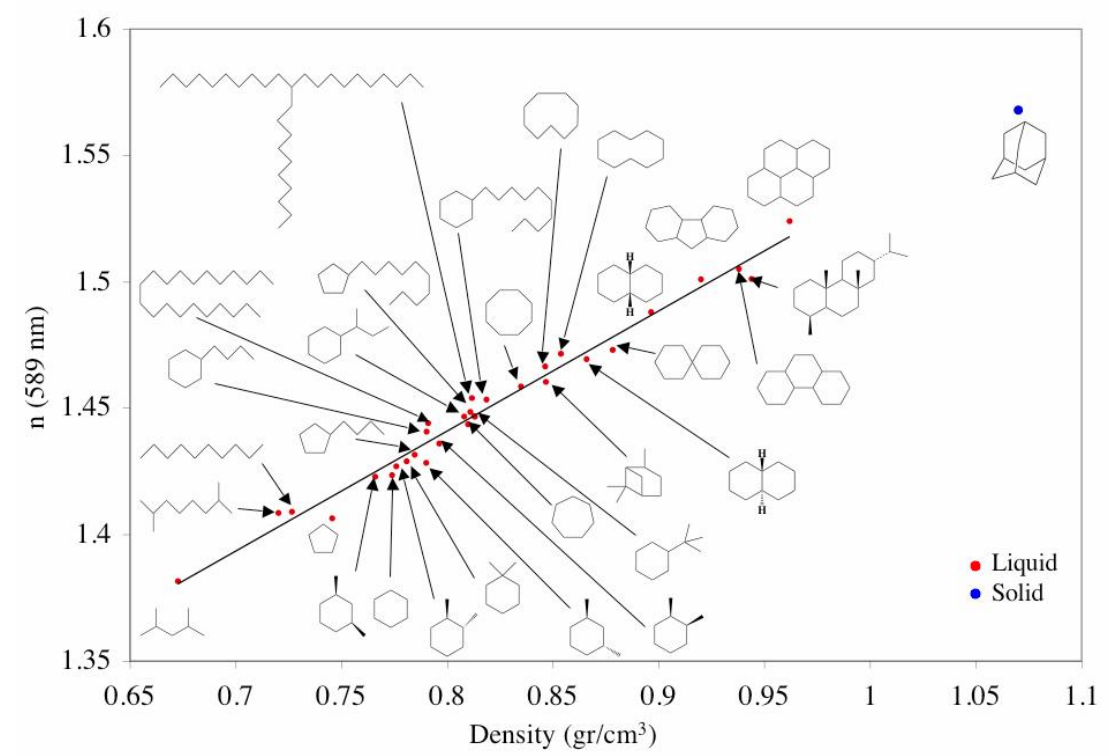

Fig. 1. Refractive index (d line) vs. density of alkanes and cycloalkanes at room temperature.

The second important conclusion from the correlation of Figure 1 is that, for comparable molecular weight, cycloalkanes possess a higher refractive index than the corresponding linear alkanes (e.g. cyclodecane, decane). Our working hypothesis interprets this feature of the correlation to mean that the supramolecular (intermolecular) organization of the cycloalkanes results in better packing and in a higher density; therefore, a higher refractive index than the equivalent molecular weight linear alkane.

A third important conclusion from the correlation of Figure 1 is that, for comparable molecular weight, the refractive index increases by increasing the number of connected rings (e.g. cyclodecane and decalin). The working hypothesis interprets this feature of the correlation to mean that polycycloalkanes are better able to minimize free volume than monocycloalkane or linear alkanes.

Finally, a fourth important conclusion of Figure 1 is that this linear relation between density and refractive index is not lost by changing from liquid to solid phase (Figure 1, adamantine solid).

But is the extrapolation of the refractive index at $589 \mathrm{~nm}$ to the refractive index at $193 \mathrm{~nm}$ valid? With the limited literature data available, ${ }^{8,9}$ we have constructed a graph of refractive index of hydrocarbons at $193 \mathrm{~nm}$ versus density (Figure 2) for comparison with the correlation of Figure 1. The linearity of the plot completely validates our hypothesis that the extrapolation of values of the refractive index at $589 \mathrm{~nm}$ is useful for predicting the values of the refractive index at $193 \mathrm{~nm}$. The excellent linear relation between density and refractive index at $193 \mathrm{~nm}$ provides confidence to extrapolate to higher densities in order to find the highest refractive index possible. Several polycycloalkanes (x's in 
Figure 2) have been synthesized or purified based on extrapolation of Figure 1, and preliminary measurements at $193 \mathrm{~nm}$ indicate that the extrapolation is justified.

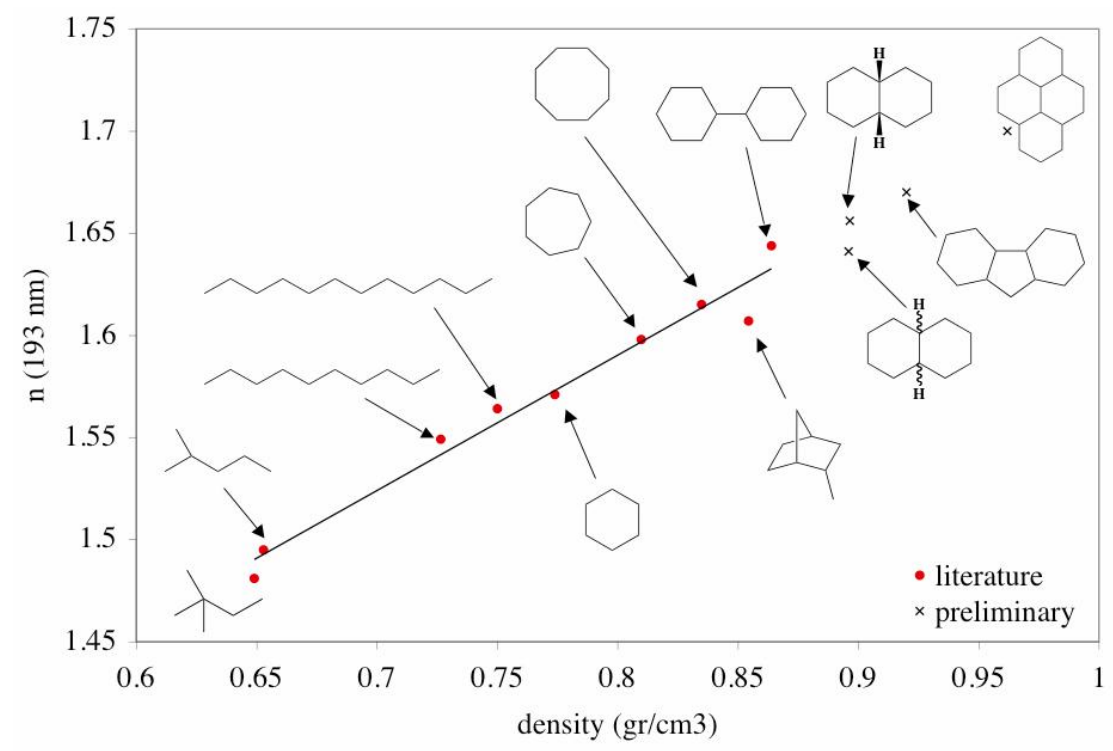

Fig. 2. Refractive index (193 nm) of cyclo- and non cyclo-alkenes as a function of the density.

In summary, from an analysis of refractive index values of alkanes and cycloalkanes at $589 \mathrm{~nm}$, it is concluded that polycycloalkanes are promising candidates for both attaining a high refractive index at $193 \mathrm{~nm}$ and a high transparency at $193 \mathrm{~nm}$. From this analysis, the following cycloalkanes were selected for initial synthesis and exhaustive purification: decalin, cyclodecane, perhydrofluorene, perhydropyrene and perhydrophenanthrene.

Except for decalin, cyclodecane and perhydrofluoene, the most promising candidates perhydropyrene and perhydrophenanthrene are not commercially available and had to be synthesized. We note that small impurities, resulting from alkane oxidation and possessing a high extinction coefficient at $193 \mathrm{~nm}$ are commonly found in commercial cycloalkanes. For example, commercially available decalin contains impurities, presumably oxidation products that absorb strongly at 193. Thus, even commercial alkanes claimed to be "highly purified" need to be subjected to exhaustive purification in order to minimize the absorption at $193 \mathrm{~nm}$. Purification techniques were developed to obtain highly pure compounds.

\section{METHODOLOGY}

\subsection{Refractive index}

For the exploratory work in the visible region, an Abbe refractometer (Bausch \& Lomb) coupled with a thermostatic bath was used. All measurements presented in this work were done at $20{ }^{\circ} \mathrm{C}$. To characterize the refractive indices at 193 nm, a Variable Angle Spectroscopic Ellipsometer (VASE, by J. A. Woollam Inc. U.S.A) was modified. The tool was originally designed to measure optical constants of thin films. It also provides the ability to measure transmission. The ellipsometer has precise $(0.044 \mathrm{mRad})$ goniometers, resulting in an accuracy of $10^{-3}$. A prism fluid cell with nominal apex angle of $45^{\circ}$ was mounted onto the sample stage. The light came into the prism cell from a monochromator. The incident angle could be adjusted by turning the sample stage. The outgoing light was collected by the detector. Additional details can be found elsewhere. ${ }^{5}$

\subsection{UV spectra}

Absorption measurements were performed in a UV-spectrometer (Shimadzu, UV-2401PC, software UVPC 3.9) on a 1 $\mathrm{mm}$ optical path cuvette. Air was used as background, and ultrapure water as reference. 


\subsection{Materials}

Decalin (Decahydronaphtalene (a cis-trans mixture) $>98 \%, 250 \mathrm{ml}$ ) was purchased from Fluka, while perhydrofluorene $(97 \%, 150517,10 \mathrm{~g})$ was purchased from Aldrich. Perhydropyrene and perhydrophenanthrene were synthesized by hydrogenation of pyrene and phenanthrene respectively. The basic physico chemical data of the cycloalkanes and polycycloalkanes used in this work are listed in Table I.

Table. 1. Structure and physical chemical properties of the cycloalkanes used in this study.

\begin{tabular}{|c|c|c|}
\hline & $\begin{array}{l}\text { Name: Cyclohexane } \\
\text { MW: } 84.09 \\
\text { Formula } \mathrm{C}_{6} \mathrm{H}_{12} \\
\text { Bp. } 80.7^{\circ} \mathrm{C} \\
\text { Density } 0.779 \mathrm{~g} / \mathrm{ml} \text { at } 25^{\circ} \mathrm{C}\end{array}$ & $\begin{array}{l}\text { Name: cyclodecane } \\
\text { MW: } 140.27 \\
\text { Formula } \mathrm{C}_{10} \mathrm{H}_{20} \\
\text { Bp. } 201{ }^{\circ} \mathrm{C} \\
\text { Density } 0.871 \mathrm{~g} / \mathrm{ml} \text { at } 25^{\circ} \mathrm{C}\end{array}$ \\
\hline & $\begin{array}{l}\text { Name: Decalin } \\
\text { MW: } 138.25 \\
\text { Formula } \mathrm{C}_{10} \mathrm{H}_{18} \\
\text { Bp. } 189-191^{\circ} \mathrm{C} \\
\text { Density } 0.896 \mathrm{~g} / \mathrm{ml} \text { at } 25^{\circ} \mathrm{C}\end{array}$ & $\begin{array}{l}\text { Name: PHF } \\
\text { MW: } 178.31 \\
\text { Formula } \mathrm{C}_{13} \mathrm{H}_{22} \\
\text { Bp. } 253^{\circ} \mathrm{C} \\
\text { Density } 0.92 \mathrm{~g} / \mathrm{ml} \text { at } 25^{\circ} \mathrm{C}\end{array}$ \\
\hline & $\begin{array}{l}\text { Name: PHP } \\
\text { MW: } 193.35 \\
\text { Formula } \mathrm{C}_{14} \mathrm{H}_{24} \\
\text { Bp. } 270^{\circ} \mathrm{C} \\
\text { Density } 0.944 \text { grml at } 25^{\circ} \mathrm{C}\end{array}$ & $\begin{array}{l}\text { Name: PHPY } \\
\text { MW: } 218.38 \\
\text { Formula } \mathrm{C}_{16} \mathrm{H}_{26} \\
\text { Bp. } 318^{\circ} \mathrm{C} \\
\text { Density } 0.96 \mathrm{~g} / \mathrm{ml} \text { at } 25^{\circ} \mathrm{C}\end{array}$ \\
\hline
\end{tabular}

\section{Purification}

\subsection{Oxidation products and elimination of impurities from commercial alkanes.}

An exhaustive purification is required even for alkanes purchased in spectral purity grade. Two main methods were used for the purification.

1) Silica gel was activated at $500{ }^{\circ} \mathrm{C}$ for 5 hours. The alkane was passed through the silica gel column. The column was prepared in a pasteur pipette and the fluid was pushed through the column by applying argon pressure on the top of the column. This method was very effective for the elimination of highly polar impurities such as oxidation products. After the chromatographic purification, the fluid was bubbled with argon or nitrogen in order to eliminate the oxygen dissolved in the fluid. It was found that an oxygen-alkane complex was formed with all alkanes, which were responsible for the high absorption at short wavelengths from 190 to $210 \mathrm{~nm}$. This complex formation will be further discussed in detail.

2) The alkane was treated with concentrated $\mathrm{H}_{2} \mathrm{SO}_{4}$ for 12 hours. The two non-miscible phases were stirred vigorously during this period. The purpose of the $\mathrm{H}_{2} \mathrm{SO}_{4}$ treatment was to eliminate unsaturated impurities (isolated double bonds) or impurities containing aromatic rings present in the fluid. After the reaction, the sulfuric acid phase was visibly yellow in color. The sulfuric acid phase was separated by decanting, the alkane layer was neutralized by treatment with $\mathrm{NaOH}$ pellets for $30 \mathrm{~min}$, and dried. A chromatography filtration through an activate carbon column was then performed to eliminate any polar impurities. Bubbling with nitrogen or argon was needed to eliminate the oxygen complex formed with the alkane. A similar method was used by Nilsson. ${ }^{10}$

The method employed should be chosen according to the nature of the impurity present in the fluid. The contaminant impurities formed from the oxidation of the fluids are highly polar and, therefore, method 1 is generally effective. 
However, if the impurity contains a $\mathrm{C}=\mathrm{C}$ double bond or aromatic system coming from the partially hydrogenated reactant, they cannot be effectively separated through the silica gel column, since the polarities of the impurities and the alkanes are similar. Therefore a treatment with sulfuric acid is needed and method 2 is more effective. The importance of the initial selection of the purification method is shown in Figure 3. Figure 3a shows the absorption spectra of commercial and purified cyclodecane using both methods; Figure $3 \mathrm{~b}$ shows the absorption spectra of commercial and purified decalin using both methods.

From Figure 3, it is obvious that the purification of cyclodecane is more effective by method number 2, while for decalin, method 1 is more effective. Impurities of decalin are mainly oxidation products of the highly reactive tertiary carbon center. These products have a high polar character and can be separated readily by silica gel chromatography. Cyclodecane is more stable against oxidation (no tertiary hydrogens) and impurities are probably residual $\mathrm{C}=\mathrm{C}$ bonds, which cannot be separated using the silica gel column from the cyclodecane because of its similar polarity. Therefore, a treatment with sulfuric acid is required to react with the $\mathrm{C}=\mathrm{C}$ bonds and to eliminate the double bond species. Oxidation products resulting from the reaction of these double bond systems with sulfuric acid are retained in the active carbon column.

\subsection{Oxygen-alkane complex formation}

The goal of the purification methods was to achieve the highest degree of transmission possible at $193 \mathrm{~nm}$, the working wavelength for the most current microlithography systems. The last step of both purification methods is the elimination of the molecular oxygen from the solution by a simple bubbling method with an inert gas (argon or nitrogen). This has been found to be a critical step, since the formation of this complex is responsible for an intense absorption band close to $193 \mathrm{~nm}$.
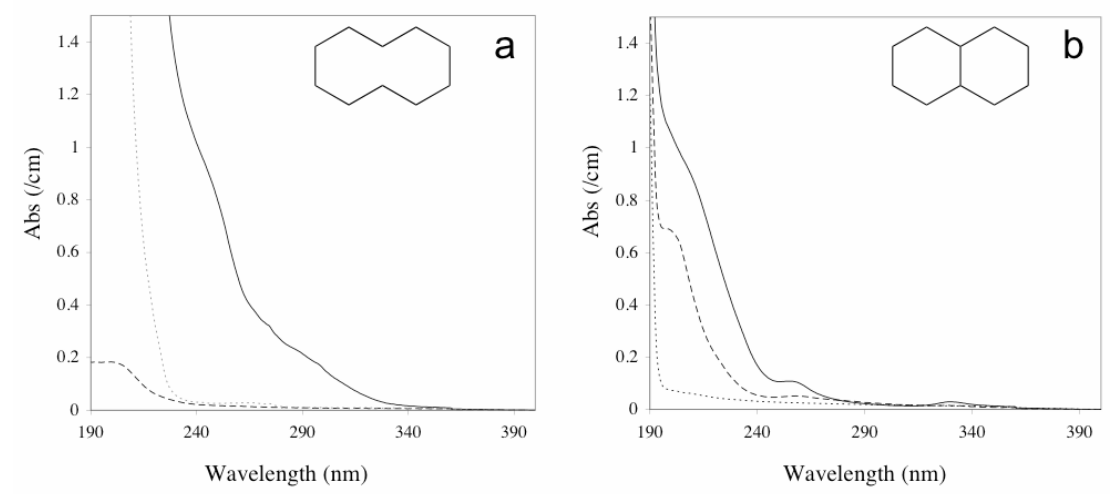

Fig. 3. Absorption spectra of cyclodecane (a) and decalin (b) commercial sample (-), after purification with method 1 ( $\cdots)$ and method $2(---)$.

\section{RESULTS}

\section{3.,10xygen-alkane complex formation}

In the present work, the interaction between molecular oxygen and linear- (pentane, octane), cyclo- (hexane, methylcyclohexane, cyclodecane) and polycyclo-alkanes (decalin, perhydrofluorene, perhydrophenanthrene, perhydropyrene) has been investigated in an attempt to delineate the origin of far UV absorption band. In all cases, a strong absorption band has been found in the region of $193 \mathrm{~nm}$. Table 2 summarizes the absorbance values for all the alkanes at $193 \mathrm{~nm}$ in the presence and absence of oxygen. A substantial decrease in the absorbance at $193 \mathrm{~nm}$ can be observed for all compounds studied when oxygen is eliminated from the media.

It is conceivable that oxygen itself may be responsible for this absorption and that no complex is formed. A control experiment was done with perfluoroalkanes, which are quite transparent at $193 \mathrm{~nm}$. Perfluoro-15-crown-5-ether and perfluoropentane were saturated with oxygen and the absorption spectrum measured. No difference could be found between the argon and oxygen saturated spectra. The result could be interpreted as due to a lower solubility of oxygen in perfluorosolvents. However, the solubility of oxygen in perfluoroalkanes is two times higher than in normal alkanes. 
Table 2. Absorbance of air and argon saturated alkanes at $193 \mathrm{~nm}$.

$$
\begin{array}{ll}
\text { Compound } & \begin{array}{c}
\text { Absorbance at } 193 \mathrm{~nm} \\
(/ \mathrm{cm}) \text { Air saturated }
\end{array}
\end{array}
$$

Absorbance at $193 \mathrm{~nm}$

(/cm) Argon saturated

\begin{tabular}{lcc}
\hline Pentane & 0.6211 & 0.19 \\
Cyclohexane & 1.2531 & 0.03 \\
Methyl-cyclohexane & $>2$ & 0.26 \\
Heptane & 1.37 & 0.04 \\
Cyclodecane & 1.1995 & 0.18 \\
Decalin & 1.1792 & 0.24 \\
Perhydrofluorene & $>2$ & $>2$ \\
Perhydrophenanthrene & $>2$ & $>2$ \\
Perhydropyrene & $>2$ & $>2$
\end{tabular}

(Molar fractions of oxygen in heptane and perfluoroheptane are $2.15 \times 10^{-3}$ and $5.5 \times 10^{-3}$ ). ${ }^{11}$ Thus, oxygen itself is not responsible for the absorption at $193 \mathrm{~nm}$ observed with alkanes. This clearly indicates that the absorption at $193 \mathrm{~nm}$ is due to the formation of the charge transfer complex between the molecular oxygen and alkanes. Evidently, the ionization potential of perfluorocarbons is too high for significant electron transfer to molecular oxygen. Therefore, no complex is formed and no absorption at $193 \mathrm{~nm}$ is observed. Both, linear and cyclic alkanes form the oxygen complex, which indicates that the latter has a non-specific "loose" molecular geometry.
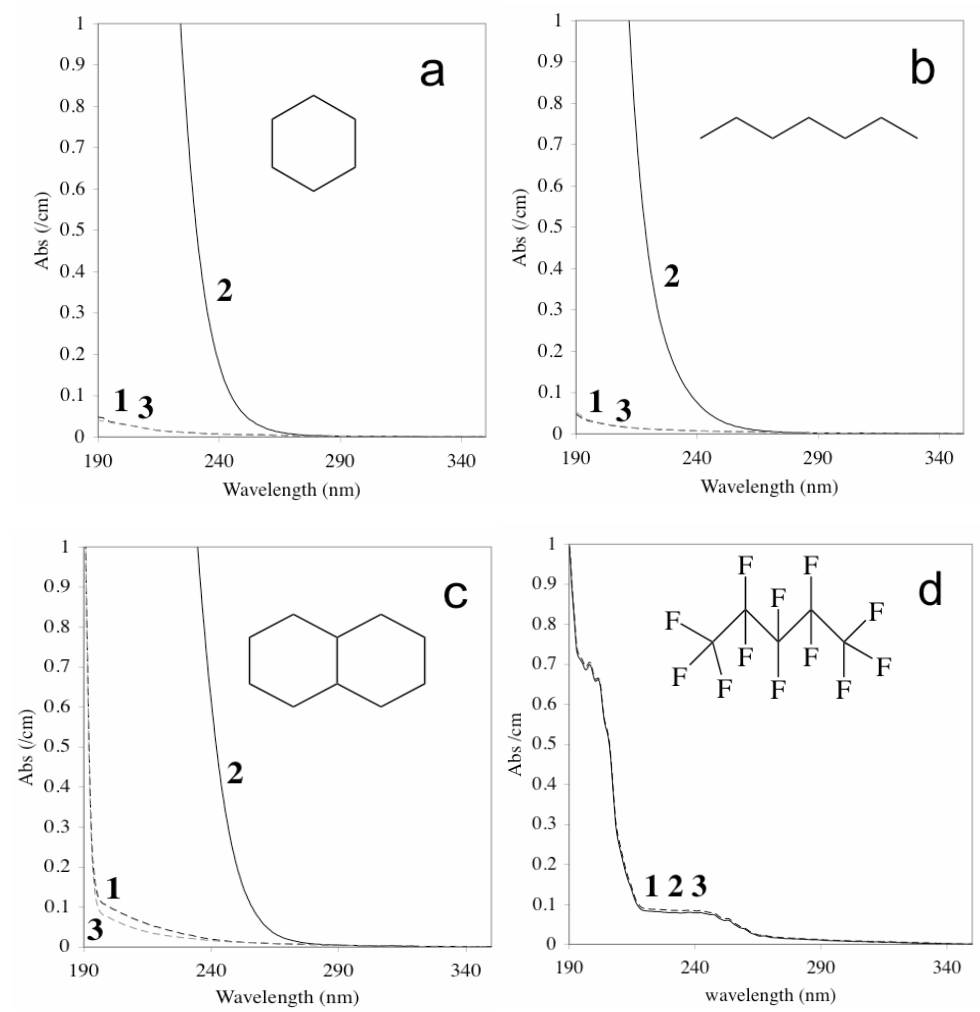

Fig. 4. Absorption spectra of argon saturated (dotted black line, 1), Oxygen saturated (black line, 2) and argon saturated again (dotted grey line, 3 ) of cyclohexane (a), heptane (b) decalin (c) and perfluoropentane (d). 
The reversibility of this complex formation was studied with several alkanes. As can be seen in Figure 4, the absorption due to the oxygen complex can be easily eliminated by bubbling argon through the liquid; the absorption reappears by bubbling molecular oxygen for a couple of minutes. Bubbling with argon reduces the absorption to the lower initial values. These experiments confirm that the observed complex is due to a weak interaction between oxygen and the organic molecule and its formation is a perfectly reversible process. From Figure 4, it can be seen that this reversibility has been observed for all types of alkanes: linear (heptane), cyclic (cyclohexane) and polycyclic (decalin). Also, the inability of the perfluorinated systems to form an oxygen complex is amply evident in (d)

\subsection{Refractive index}

The experimental refractive index of the polycycloalkanes as a function of wavelength is presented in Figure 5. As can be observed, the eventual value of refractive index at $193 \mathrm{~nm}$ follows the same trend as the value at $589 \mathrm{~nm}$. PHPY is the compound with the higher refractive index at $193 \mathrm{~nm}(\mathrm{n} 193 \mathrm{~nm}=1.7014)$, followed by PHF $\left(\mathrm{n}_{193 \mathrm{~nm}}=1.6684\right)$, Decalin $\left(\mathrm{n}_{193 \mathrm{~nm}}=1.6238\right)$ and cyclodecane $\left(\mathrm{n}_{193 \mathrm{~nm}}=1.6211\right)$ and cyclohexane $\left(\mathrm{n}_{193 \mathrm{~nm}}=1.5618\right)$. R. French et al. measured similar values, ${ }^{8} 1.571$ and 1.641 , for cyclohexane and decalin respectively. This result confirms the validity of the preliminary study performed with the literature data at $589 \mathrm{~nm}^{7}$

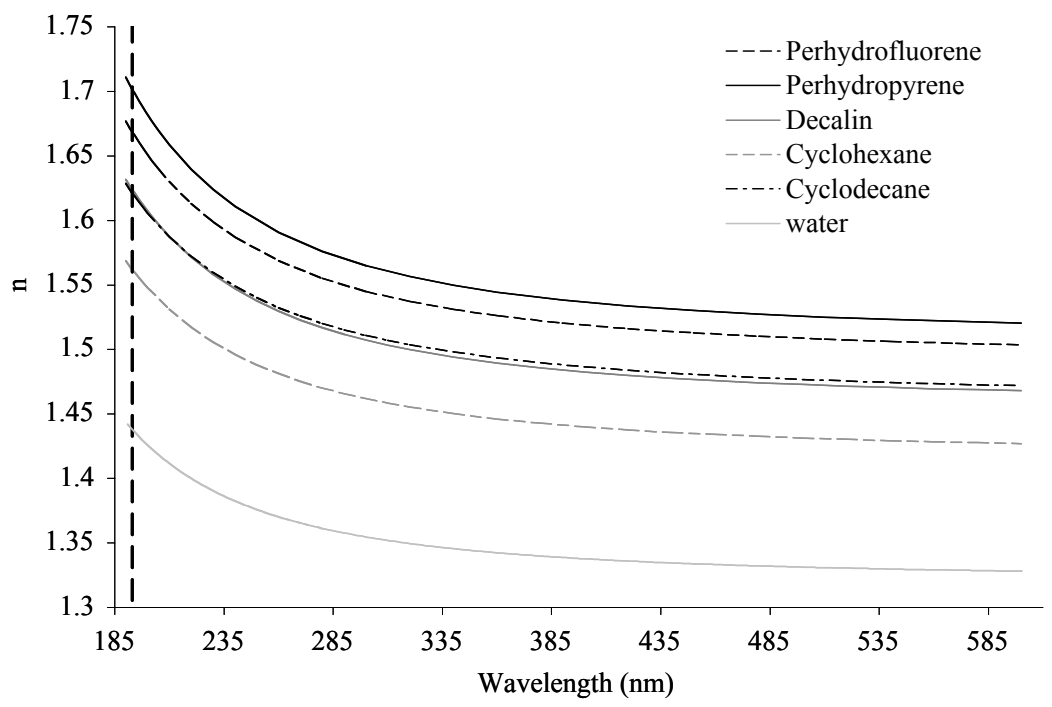

Fig. 5. Refractive index (d line) vs. density of alkanes and cycloalkanes at room temperature.

\subsection{Absorption Spectra}

An absorbance lower than $0.15 / \mathrm{cm}$ is specified as a requierement for a second and third generation of immersions fluids. Alkanes were selected as candidates for immersion fluids because of their low absorption due to the absence of $\mathrm{n}$ and $\pi$ molecular orbitals and therefore, the corresponding low energy electronic transitions in the deep UV. Absorption spectra of commercial alkanes and cycloalkanes were measured, and an unexpected strong absorption was observed in all cases, showing the need for purification. In addition, strong contribution to the absorption has been attributed to the formation of a reversible charge-transfer complex between the cycloalkane and molecular oxygen.

The absorption spectra of all candidates after purification and purging with argon or nitrogen are shown in Figure 6. As can be observed, some of the candidates present a high absorption at $193 \mathrm{~nm}$ even after removal of the dissolved oxygen. Cyclohexane does not present almost any absorption at $193 \mathrm{~nm}$. However, decalin and cyclodecane have an acceptable absorbance lower than $0.2 / \mathrm{cm}$ at $193 \mathrm{~nm}$, while PHPY, PHP and PHF have an unacceptable high absorption at $193 \mathrm{~nm}$. In spite of several purification processes, some impurities maybe present in the fluids. The small absorption band with a maximum at $260 \mathrm{~nm}$ maybe attributed to impurities which have made it through the exhaustive purification process. These traces of some absorbing compound can contribute to the higher absorption at $193 \mathrm{~nm}$. Therefore, it is still 
possible that further purification and elimination of those traces may decrease the absorption at the wavelength of interest $(193 \mathrm{~nm})$.

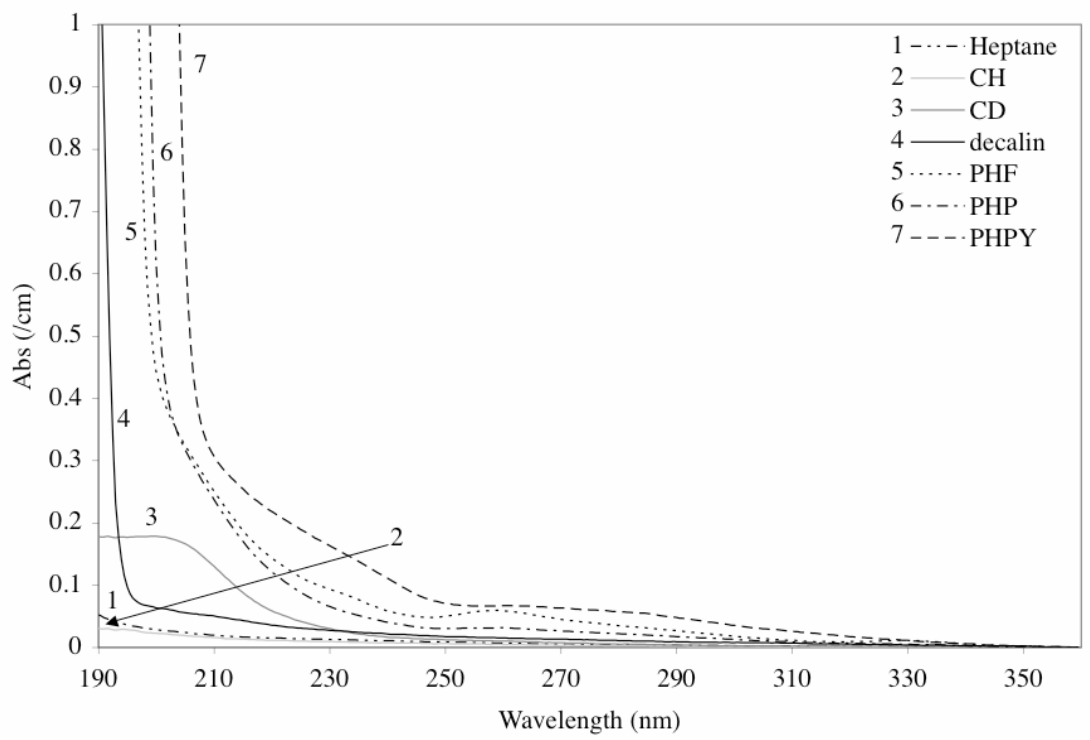

Fig. 6. Absorption spectra after purification of cycloalkanes and polycycloalkanes used in this study.

\subsection{Exposure results and analysis}

A JSR2928 resist (54nm) was used for exposure, coated over an AR layer (AR29-A BARC 39nm) with a JSR TCX041 topcoat $(40 \mathrm{~nm})$. The film stack was optimized by using IL-SIM software.11 Decalin and cyclodecane were chosen as immersion fluids because of their high refractive index (decalin $\mathrm{n} 193 \mathrm{~nm}=1.6238)$ and acceptable transparency at 193 $\mathrm{nm}$. The SEM pictures are shown in Figure 7 and 8 . Resolution of $33 \mathrm{~nm}$ and $32 \mathrm{~nm}$ has been demonstrated using effective NA of 1.45 and 1.50 when decalin was used as an immersion fluid (Figure 7). The modulation is high and the photoresist is not degraded by the fluids.

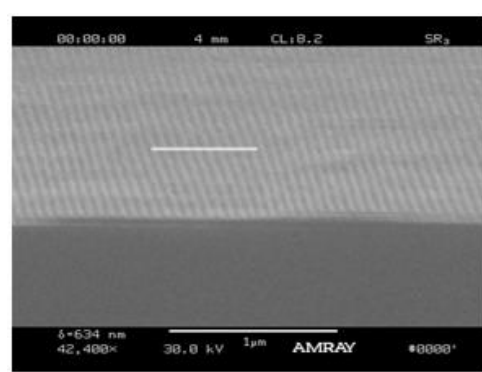

1.5 NA. $32 \mathrm{~nm}$ half pitch

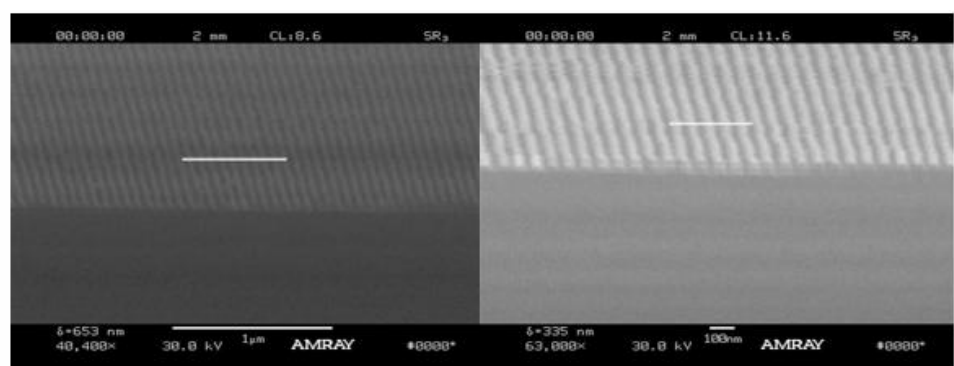

$1.45 \mathrm{NA} .33 \mathrm{~nm}$ half pitch

Fig. 7. Imaging with Decalin.

In the case of the cycloedecane a low modulation was found probably because of the high absorption of the fluid. Some chemical interaction between the photoresit and the cyclodecane cannot be excluded. Further studies on this issue will be performed. 


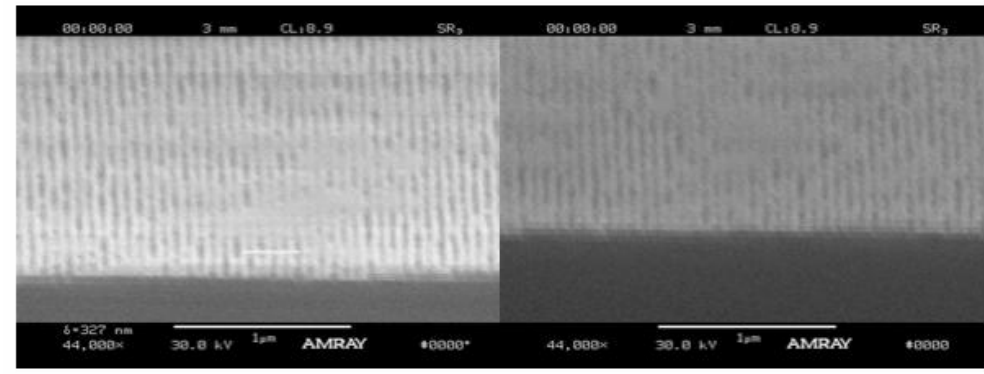

1.45 NA. $33 \mathrm{~nm}$ half pitch

Fig. 8. Imaging with Cyclodecane

\section{CONCLUSION}

Cycloalkanes and polycycloalkanes have been studied as possible candidates for immersion fluids at $193 \mathrm{~nm}$. The refractive index of cycloalkanes and polycycloalkanes increase with increasing density of the fluid. In terms of structure, the refractive index of the fluid has been observed to increase by increasing the number of transoid-connected cyclohexane rings.

Two simple alkane purification methods were developed and standardized, which led to high transparent fluids in the VUV region. There has been special interest in the absorption of these alkanes at 193 nm since alkanes have been proposed as second generation immersion fluids for photolithography printing processes in microelectronics. Alkanes and cycloakkanes have been purified until the lowest absorption in the VUV near $193 \mathrm{~nm}$ is produced. Purification of alkanes is a critical stage in the processes of selection and characterization of possible candidates as immersion fluids. A non purified candidate should be eliminated due to its high absorption coming from some traces of impurities, which could falsely be attributed to the organic compound itself.

An important point in the purification process is the elimination of oxygen from the fluid. A complex formation between the oxygen and the organic compound leads to an unexpected high absorbance of all alkanes studied below $240 \mathrm{~nm}$. However, after a simple bubbling of argon or nitrogen through the fluid, the absorption is generally reduced demonstrating the weak character of this complex and its reversibility.

In terms of absorption the increasing number of transoid-connected cyclohexane rings have been observed to lower energy gap between the HOMO and LUMO, which causes the absorption band to shift to wavelengths longer than 193 $\mathrm{nm}$. However, decalin and cyclodecane bear the potential to function acceptably as a second-generation fluid (n193nm $>1.6 \mathrm{~nm}$, Absorption $<0.15 / \mathrm{cm}$ ). The resolution of $193 \mathrm{~nm}$ immersion lithography has been pushed down to $32 \mathrm{~nm}$ using decalin and cyclodecane as immersion fluids. With the latter, low modulation problems were found during exposure because of high absorption or possible chemical interaction between the photoresist and the fluid.

\section{REFERENCES}

1. S. Owa and H. Nagasaka, "Advantage and feasibility of immersion lithography", J. Microlith., Microfab. and Microsys. 3(1), 97-103 (2004).

2. J. Mulkens, D. G. Flagello, B. Streefkerk, P. Graupner, "Benefits and limitations of immersion lithography", J. Microlith., Microfab. and Microsys. 3(1), 104-114 (2004).

3. B. W. Smith, A. Bourov, H. Kang, F. Cropanese, Y. Fan, N. Lafferty, L. Zavyalova, "Water immersion optical lithography at $193 \mathrm{~nm}$ " J. Microlith., Microfab. and Microsy.s 3(1), 44-51 (2004).

4. Budhlall, B.; Parris, G.; Zhang, P.; Gao, X.; Zarkov, Z.; Ross, B., Proc. SPIE 2005, 5754, 622. B. Budhlall, G. Parris, P. Zhang, X. Gao, Z. Zarkov and B. Ross, "High Refractive Index Immersion Fluids for $193 \mathrm{~nm}$ Immersion Lithography“, SPIE Proc. 5754, 622-629, (2005).

5. J. Zhou, Y. Fan, A. Bourov, N. Lafferty, F. Cropanese, L. Zavyalova, A. Estroff, B. W. Smith, "Immersion lithography fluids for high NA 193 nm lithography“ SPIE Proc. 5754, 630-637, (2005). 
6. S. Pemg, R. H. French, W. Qiu, R. C. Wheland, M. Yang, M. F. Lemon, M. K. Crawford, "Second Generation fluids for $193 \mathrm{~nm}$ Immersion Lithography“ SPIE Proc. 5754, 427-434 (2005).

7. D. R. Lide, 85th edition Handbook of Chemistry and Physics, CRC Press, 2004.

8. P. Zhang, Patent Appl. US2005/0173682 A1.

9. R. French, Patent WO 2005/119371 A1.

10. O. Nilsson "On the Preparation of UV Transparent, Saturated Hydrocarbons" Acta Chem. Scan., 21, 1501-1506, 1967.

11. "Solubility Data Series, volume 7 , Oxygen and Ozone" Ed. R. Battino, Pergamon Press Great Britain, 1981. 